Article

\title{
Associations of Serum Manganese Levels with Prediabetes and Diabetes among $\geq 60$-Year-Old Chinese Adults: A Population-Based Cross-Sectional Analysis
}

\author{
Xuan Wang ${ }^{1}$, Mingyue Zhang ${ }^{2}$, Guang Lui ${ }^{2}$, Hong Chang ${ }^{3}$, Meilin Zhang ${ }^{1}$, Wei Liu ${ }^{4}$, \\ Ziwei $\mathrm{Li}^{1}{ }^{1}$, Yixin Liu ${ }^{1}$ and Guowei Huang ${ }^{1, *}$ \\ 1 Department of Nutrition and Food Science, School of Public Health, Tianjin Medical University, \\ 22 Qixiangtai Road, Heping District, Tianjin 300070, China; wangxuan@tmu.edu.cn (X.W.); \\ defjmmm@163.com (M.Z.); lzw30706sky@163.com (Z.L.); tjmulyx@126.com (Y.L.) \\ 2 Department of Physics and Chemistry, Tianjin Centers for Disease Control and Prevention, 6 Huayue Road, \\ Hedong District, Tianjin 300070, China; zhmyue@163.com (M.Z.); lvguang2003@163.com (G.L.) \\ 3 Department of Rehabilitation and Sports Medicine, Tianjin Medical University, 22 Qixiangtai Road, \\ Heping District, Tianjin 300070, China; changhong@tmu.edu.cn \\ 4 Community Health Service Center for Shuangjie Town of Beichen District in Tianjin, Zhangwan Road, \\ Shuangjie Town, Beichen District, Tianjin 300400, China; liuweitmu@126.com \\ * Correspondence: huangguowei@tmu.edu.cn; Tel.: +86-22-8333-6606; Fax: +86-22-8333-6603
}

Received: 20 May 2016; Accepted: 8 August 2016; Published: 13 August 2016

\begin{abstract}
Older adults can experience glucose metabolism dysfunction, and although manganese may help regulate glucose metabolism, there is little information regarding this association among older people. This cross-sectional study included 2402 Chinese adults who were $\geq 60$ years old in 2013 (Tianjin, China), and evaluated the associations of serum manganese with prediabetes and diabetes. Serum manganese levels were measured using inductively coupled plasma mass spectrometry. Multivariable logistic regression models were used to evaluate the sex-specific associations of manganese levels with diabetes and prediabetes after adjusting for confounding factors (age, sex, life style factors, and health status). Based on the WHO criteria, prediabetes was observed in $15.1 \%$ of men and $13.4 \%$ of women, while diabetes was observed in $30.0 \%$ of men and $34.4 \%$ of women. In the final model, the odds ratios (95\% confidence interval) for prediabetes according to manganese quartile were 1.000, $0.463(0.269-0.798), 0.639(0.383-1.065)$, and $0.614(0.365-1.031)$ among men and 1.000, $0.773(0.498-1.200), 0.602(0.382-0.947)$, and $0.603(0.381-0.953)$ among women $(p$ for trend $=0.134$ and 0.015 , respectively). The lowest prevalence of diabetes among men occurred at a moderate range of serum manganese $(p<0.05)$. Therefore, appropriate serum manganese levels may help prevent and control prediabetes and diabetes.
\end{abstract}

Keywords: manganese; prediabetes; diabetes; older adult

\section{Introduction}

Diabetes mellitus is a major public health problem [1] and a leading cause of morbidity and mortality, with an estimated 346 million adults being affected worldwide in 2011 [2]. In China, the prevalence of diabetes is estimated to be $11.6 \%$ among adults [3], and the number and proportion of older adults are rapidly increasing in China and throughout the world [4]. The prevalence of diabetes also increases with age, and reaches $22.5 \%$ among adults who are $\geq 60$ years old [3].

Trace elements, such as copper, zinc, iron, selenium, and manganese (Mn), are essential for human health and are involved in various metabolic and biological functions [5]. Deficiencies or excesses in 
these trace elements are usually related to human diseases. Mn is an essential trace element that is involved in normal immune functions, regulation of blood sugar and cellular energy, and protection against free radicals [6,7]. Mn is also a cofactor for several enzymatic systems and is required for normal insulin synthesis and secretion [8,9]. Moreover, some trace elements (e.g., Mn) can potentiate insulin's action in reducing blood glucose levels [10]. However, it remains unclear whether Mn has effects in patients with diabetes.

Several studies have reported direct associations of Mn levels with diabetes [11-17], although it remains unclear whether Mn plays a positive or negative role. Furthermore, no studies have assessed the relationship between Mn levels and diabetes among seniors. The older age of this population also increases the risk of nutrient deficiencies, which can quickly lead to undernutrition and possibly contribute to the development of diseases [18]. Therefore, it is important to study the relationship between diabetes and Mn levels among older adults.

Prediabetes often precedes diabetes and is characterized by impaired fasting glucose (IFG) and/or impaired glucose tolerance (IGT) [19]. Prediabetes is also especially prevalent among older adults [4,20]. Compared to individuals with normal glucose metabolism, patients with prediabetes have a 5-15-fold higher risk of developing type 2 diabetes [21]. However, prediabetes is a reversible state [22], and the identification of risk factors for this state is an important step for the early prevention of diabetes. To the best of our knowledge, no studies have investigated the relationship between serum Mn levels and prediabetes among community-dwelling older adults. Therefore, we performed a cross-sectional study to determine whether serum Mn levels were associated with prediabetes and diabetes among community-dwelling $\geq 60$-year-old adults.

\section{Materials and Methods}

\subsection{Study Population}

This study included 3066 individuals who were $\geq 60$ years old, living in the Shuangjie community of the Beichen district, and underwent health check-ups in 2013 at the Community Health Service Center (Beichen district, Tianjin, China). We invited all of these individuals to participate in a geriatric assessment, which included medical status, physical function, and cognitive function, and 2623 of the individuals completed the assessment (response rate: 85.6\%). We excluded individuals with incomplete data $(n=178)$ and individuals whose serum Mn levels had not been measured $(n=43)$. Therefore, the final study population included 2402 participants (mean age: $67.5 \pm 5.4$ years; $40.1 \%$ men). The study's protocol was approved by the Ethics Committee of Tianjin Medical University (TMUhMEC20120110), and all participants provided their written informed consent.

\subsection{Serum Mn Measurements}

Venous blood samples were collected after an overnight fast, were allowed to clot at room temperature, and were then centrifuged at $1000 \mathrm{rpm}$ for $10 \mathrm{~min}$. The serum was then transferred to Eppendorf tubes and stored at $-20{ }^{\circ} \mathrm{C}$ until the analysis. Serum Mn levels were measured at the Tianjin Center for Disease Control and Prevention (Tianjin, China), based on a standardized protocol that incorporated sample processing, storage, and transportation to the analysis center. Serum Mn levels were analyzed using inductively coupled plasma mass spectrometry (Agilent7500cx; AGILENT TECHNOLOGIES INC., Santa Clara, CA, USA). The lower limit of detection for this assay was $0.035 \mu \mathrm{g} / \mathrm{L}$, and scandium was used as the standard reference material for internal quality assurance and control (AGILENT TECHNOLOGIES INC). The inter-assay coefficients of variation ranged from $0.95 \%$ to $3.4 \%$ for serum $\mathrm{Mn}$ samples.

\subsection{Biochemical Assessments}

Levels of fasting plasma glucose (FPG), total cholesterol (TC), triglycerides (TG), creatinine (CRE), and blood urea nitrogen (BUN) were determined using the Roche Modular P system 
(Roche Diagnostic Company, Switzerland). The creatinine clearance rate was calculated as $((140-$ age in years $) \times$ weight in $\mathrm{kg}) /(72 \times \mathrm{CRE}$ in $\mathrm{mg} / \mathrm{mL})$, and $15 \%$ was subtracted from the calculated value for women.

\subsection{Assessment of Diabetes and Prediabetes}

Diabetes was defined by a self-reported physician's diagnosis, use of hypoglycemic medication, or insulin treatment at the time of the interview. Among undiagnosed participants, diabetes was defined as FPG levels of $\geq 126 \mathrm{mg} / \mathrm{dL}(\geq 7.0 \mathrm{mmol} / \mathrm{L})$ and prediabetes was defined as FPG levels of 110-126 mg/dL (6.1-7.0 mmol/L), based on the 1999 World Health Organization diagnostic criteria [23]. In this study, dysglycemia was defined as cases of both diabetes and prediabetes [24].

\subsection{Assessment of Other Variables}

The participants' anthropometric characteristics (height and body weight) were recorded using a standardized protocol. Body mass index (BMI) was calculated as weight $/$ height ${ }^{2}\left(\mathrm{~kg} / \mathrm{m}^{2}\right)$. A history of diabetes, hypertension, stroke, and/or coronary heart disease (CHD) was identified based on the participants' responses regarding a physician's diagnosis, taking any corresponding medication(s), or other treatment (current or previous). Information regarding smoking status (never, former smoker, and current smoker) and drinking status (never, daily drinker, and occasional drinker) were obtained using a single choice question questionnaire. Exercise status was assessed using a single self-reported question regarding frequency and duration of exercise during the last year: (1) "high" was defined as $\geq 3$ sessions of $\geq 30$ min each week; (2) "low" was defined as some exercise during the past year that did not qualify as "high"; and (3) "none" was defined as no exercise.

\subsection{Statistical Analysis}

Tests were performed to analyze the interactions between the serum Mn levels and sex in the final models. No significance interactions were found ( $p$ for interaction $>0.05$ ). However, some studies have suggested that there are sex-specific differences in the homeostatic mechanisms that regulate blood $\mathrm{Mn}$ levels $[25,26]$. Furthermore, there are sex difference in the development, prevention, and management of diabetes [27-29]. Therefore, we categorized the participants according to sex for the reported of the results. The participants' clinical and biochemical data were presented as mean \pm standard deviation, number $(\%)$, or median and interquartile range for variables with a skewed distribution. Differences among the dysglycemia groups were evaluated using analysis of variance for continuous variables or using the $\chi^{2}$ test for categorical variables. Logistic regression analysis was used to determine the odds ratios (ORs) and 95\% confidence intervals (CI) for the associations of serum Mn levels with prediabetes or diabetes. The ORs were calculated using a crude model and two adjusted models (Model A: adjusted for age, BMI, smoking status, drinking status, and exercise status; Model B: further adjusted for blood pressure, TG levels, TC levels, duration of diabetes, antidiabetic medication use, and a history of hypertension, cardiovascular disease, and/or stroke). Interactions between the serum Mn levels and sex were tested by the addition of the cross-product terms in the regression model. $p$-values for linear trends were calculated using the Mn level quartiles, and results were considered statistically significant at a two-tailed $p$-value of $<0.05$. All statistical analyses were performed using SPSS software (version 13.0; SPSS Inc., Chicago, IL, USA).

\section{Results}

Based on the World Health Organization criteria, we identified prediabetes among $14.1 \%$ of the participants (339/2402; men: 15.1\% (146/964), women: 13.4\% (193/1438)), and diabetes among 32.6\% of the participants (783/2402; men: 30.0\% (289/964), women: 34.4\% (494/1438)). Serum Mn levels ranged from $0 \mu \mathrm{g} / \mathrm{L}$ to $46.34 \mu \mathrm{g} / \mathrm{L}$, with an arithmetic mean of $9.49 \mu \mathrm{g} / \mathrm{L}$ and a geometric mean of $5.03 \mu \mathrm{g} / \mathrm{L}$. The serum Mn concentrations were $2.91 \mu \mathrm{g} / \mathrm{L}, 5.79 \mu \mathrm{g} / \mathrm{L}$, and $11.13 \mu \mathrm{g} / \mathrm{L}$ for the 25th, 50th, and 75th percentiles, respectively (Table 1). 
Table 1. Descriptive participants' characteristics by sex.

\begin{tabular}{|c|c|c|c|c|}
\hline Variables & Total & Men & Women & $p$-Value \\
\hline$N$ & 2402 & 964 & 1438 & \\
\hline Age, year & $67.54 \pm 5.40$ & $67.49 \pm 5.41$ & $67.58 \pm 5.38$ & 0.684 \\
\hline $\mathrm{SBP}, \mathrm{mmHg}$ & $136.68 \pm 16.57$ & $137.54 \pm 16.99$ & $136.10 \pm 16.26$ & 0.037 \\
\hline $\mathrm{DBP}, \mathrm{mmHg}$ & $82.06 \pm 8.82$ & $83.01 \pm 9.64$ & $81.42 \pm 8.17$ & $<0.001$ \\
\hline BMI, $\mathrm{kg} / \mathrm{m}^{2}$ & $26.42 \pm 3.54$ & $26.29 \pm 3.28$ & $26.51 \pm 3.71$ & 0.134 \\
\hline $\begin{array}{l}\text { Blood index } \\
\text { TC, mmol/L } \\
\text { TG, } \mathrm{mmol} / \mathrm{L} \\
\text { FPG, } \mathrm{mmol} / \mathrm{L} \\
\mathrm{BUN}, \mathrm{mmol} / \mathrm{L} \\
\mathrm{CCr}, \mathrm{min} / \mathrm{L} \\
\mathrm{CRE}, \mu \mathrm{mol} / \mathrm{L}\end{array}$ & $\begin{array}{c}5.37 \pm 1.03 \\
1.51(1.10,2.10) \\
6.5 \pm 11.90 \\
5.81 \pm 1.63 \\
60.14 \pm 22.69 \\
97.64 \pm 34.04\end{array}$ & $\begin{array}{c}5.05 \pm 0.93 \\
1.40(1.00,1.96) \\
6.49 \pm 1.77 \\
6.01 \pm 1.61 \\
64.51 \pm 16.03 \\
108.23 \pm 44.80\end{array}$ & $\begin{array}{c}5.59 \pm 1.04 \\
1.60(1.20,2.20) \\
6.53 \pm 1.98 \\
5.68 \pm 1.66 \\
57.21 \pm 25.82 \\
90.54 \pm 21.58\end{array}$ & $\begin{array}{c}<0.001 \\
<0.001 \\
0.587 \\
<0.001 \\
<0.001 \\
<0.002\end{array}$ \\
\hline $\begin{array}{l}\text { Smoking status, } \%(n) \\
\text { Current smoker } \\
\text { Former smoker } \\
\text { Nonsmoker }\end{array}$ & $\begin{array}{c}19.2(462) \\
3.5(84) \\
77.3(1856)\end{array}$ & $\begin{array}{c}32.0(308) \\
6.0(58) \\
62.0(598)\end{array}$ & $\begin{array}{c}10.7(154) \\
1.8(26) \\
87.5(1258)\end{array}$ & $\begin{array}{l}<0.001 \\
<0.001 \\
<0.001\end{array}$ \\
\hline $\begin{array}{l}\text { Drinking status, } \%(n) \\
\text { Daily drinker } \\
\text { Occasional drinker } \\
\text { Nondrinker }\end{array}$ & $\begin{array}{c}7.1(170) \\
4.8(116) \\
88.1(2116)\end{array}$ & $\begin{array}{l}16.7(161) \\
11.4(110) \\
71.9(693)\end{array}$ & $\begin{array}{c}0.6(9) \\
0.4(6) \\
99.0(1423)\end{array}$ & $\begin{array}{l}<0.001 \\
<0.001 \\
<0.001\end{array}$ \\
\hline $\begin{array}{c}\text { Exercise status, \% (n) } \\
\text { High } \\
\text { Low } \\
\text { None }\end{array}$ & $\begin{array}{l}51.2(1229) \\
16.5(396) \\
32.3(777)\end{array}$ & $\begin{array}{l}55.7(537) \\
14.4(139) \\
29.9(288)\end{array}$ & $\begin{array}{l}48.1(692) \\
17.9(257) \\
34.0(489)\end{array}$ & $\begin{array}{l}0.001 \\
0.029 \\
0.038\end{array}$ \\
\hline Serum $M n, \mu g / L$ & $5.79(2.91,11.13)$ & $6.02(2.86,11.17)$ & $5.64(2.95,11.10)$ & 0.694 \\
\hline $\begin{array}{c}\text { History of Disease } \\
\text { Obesity, \% }(n) \\
\text { Diabetes, \% }(n) \\
\text { Hypertension, \% }(n) \\
\text { Stroke, \% }(n) \\
\text { CHD, \% }(n)\end{array}$ & $\begin{array}{c}29.8(715) \\
28.4(682) \\
72.5(1742) \\
4.6(111) \\
14.7(353)\end{array}$ & $\begin{array}{c}28.4(274) \\
25.6(247) \\
72.6(700) \\
7.1(68) \\
10.9(105)\end{array}$ & $\begin{array}{c}30.7(441) \\
30.3(435) \\
72.5(1042) \\
3.0(43) \\
17.2(248)\end{array}$ & $\begin{array}{c}0.238 \\
0.014 \\
0.972 \\
<0.001 \\
<0.001\end{array}$ \\
\hline
\end{tabular}

Abbreviations: SBP, systolic blood pressure; DBP, diastolic blood pressure; BMI, body mass index; TC, total cholesterol; TG, triglyceride; FPG, fasting plasma glucose; BUN, blood urea nitrogen; $\mathrm{CCr}$, creatinine clearance; CRE, creatinine; CHD, coronary heart disease; Mn, manganese; $p$ values were calculated from Chi-square test for categorical variables and Student's $t$ tests or the Wilcoxon rank-sum test were used for between-group comparisons of normally distributed and skewed data, respectively; All continuous variables were presented as means $\pm \mathrm{SD}$ or medians (interquartile ranges), and all categorical variables were presented as number (proportions).

\subsection{Participant Characteristics}

The participants' characteristics according to sex are shown in Table 1. There were no sex-specific differences in age, BMI, FPG levels, serum Mn levels, or the prevalences of obesity and hypertension. Men had significantly higher values for blood pressure, BUN, and CRE, and significantly lower levels of TC and TG $(p<0.05)$. Men also exhibited higher prevalences of stroke, current smoker status, daily drinker status, and higher exercise status, while women exhibited a higher prevalence of diabetes and CHD.

The participants' characteristics according to dysglycemia category are presented in Table 2. Participants with diabetes or prediabetes were more likely than normoglycemic participants to have higher values for blood pressure and BMI. In addition, men with prediabetes or diabetes had a higher prevalence of hypertension, while women exhibited higher values for systolic blood pressure, BMI, TG levels, hypertension, and obesity. Furthermore, older men with diabetes and older women with prediabetes were more likely to have exercised more during the last year, compared to normoglycemic participants (men: $p<0.001$; women: $p=0.045$ ). Men with diabetes were also more likely to have exercised more (vs. men with prediabetes), although the opposite trend was observed for women. Normoglycemic participants were significantly less likely to do any exercise, compared to participants with prediabetes or diabetes $(p<0.001)$. Women with prediabetes had lower serum Mn levels, compared to women with normoglycemia or diabetes $(p=0.001)$. 
Table 2. Participants' characteristics according to sex and categories of dysglycemia.

\begin{tabular}{|c|c|c|c|c|c|c|c|c|}
\hline \multirow[b]{2}{*}{ Variables } & \multicolumn{4}{|c|}{ Men } & \multicolumn{4}{|c|}{ Women } \\
\hline & $\begin{array}{l}\text { Normoglycemia } \\
\quad(n=529)\end{array}$ & $\begin{array}{l}\text { Prediabetes } \\
\quad(n=146)\end{array}$ & $\begin{array}{l}\text { Diabetes } \\
(n=289)\end{array}$ & $p$-Value & $\begin{array}{c}\text { Normoglycemia } \\
\quad(n=751)\end{array}$ & $\begin{array}{l}\text { Prediabetes } \\
\quad(n=193)\end{array}$ & $\begin{array}{l}\text { Diabetes } \\
(n=494)\end{array}$ & $p$-Value \\
\hline Age, year & $67.41 \pm 5.41$ & $66.97 \pm 5.24$ & $67.90 \pm 5.51$ & 0.208 & $67.29 \pm 5.05$ & $67.27 \pm 5.23$ & $68.14 \pm 5.87$ & 0.106 \\
\hline $\mathrm{SBP}, \mathrm{mmHg}$ & $136.00 \pm 17.24$ & $141.85 \pm 8.64$ * & $138.19 \pm 15.23^{\#}$ & 0.001 & $134.44 \pm 15.93$ & $137.54 \pm 17.33$ * & $138.07 \pm 16.08$ * & $<0.001$ \\
\hline DBP, $\mathrm{mmHg}$ & $82.99 \pm 9.71$ & $84.97 \pm 10.00 *$ & $82.03 \pm 9.18^{\#}$ & 0.011 & $81.10 \pm 8.05$ & $82.17 \pm 8.70$ & $81.49 \pm 8.14$ & 0.325 \\
\hline BMI, $\mathrm{kg} / \mathrm{m}^{2}$ & $26.04 \pm 3.36$ & $26.62 \pm 3.33$ & $26.59 \pm 3.07^{*}$ & 0.03 & $26.09 \pm 3.64$ & $26.96 \pm 4.04^{*}$ & $26.96 \pm 3.61 *$ & $<0.001$ \\
\hline $\begin{array}{c}\text { Blood index } \\
\text { TC, mmol/L } \\
\text { TG, mmol/L } \\
\text { FPG, mmol/L } \\
\text { BUN, } \mathrm{mmol} / \mathrm{L} \\
\text { CRE, } \mu \mathrm{mol} / \mathrm{L}\end{array}$ & $\begin{array}{c}5.01 \pm 0.91 \\
1.37(1.00,1.90) \\
5.47 \pm 0.43 \\
5.97 \pm 1.53 \\
107.96 \pm 46.23\end{array}$ & $\begin{array}{c}5.15 \pm 0.92 \\
1.48(1.07,2.10) \\
6.43 \pm 0.26^{*} \\
5.97 \pm 1.60 \\
107.01 \pm 16.29\end{array}$ & $\begin{array}{c}5.08 \pm 0.95 \\
1.45(1.05,2.00) \\
8.38 \pm 2.14^{*, \#} \\
6.12 \pm 1.74 \\
109.35 \pm 51.53\end{array}$ & $\begin{array}{c}0.214 \\
0.087 \\
<0.001 \\
0.414 \\
0.858\end{array}$ & $\begin{array}{c}5.59 \pm 1.01 \\
1.59(1.20,2.10) \\
5.43 \pm 0.44 \\
5.61 \pm 1.49 \\
89.48 \pm 14.63\end{array}$ & $\begin{array}{c}5.71 \pm 1.03 \\
1.71(1.30,2.41)^{*} \\
6.43 \pm 0.26^{*} \\
5.64 \pm 1.71 \\
90.39 \pm 15.90\end{array}$ & $\begin{array}{c}5.54 \pm 1.09 \\
1.66(1.20,2.30) \\
8.24 \pm 2.511^{*}, \# \\
5.78 \pm 1.80 \\
92.22 \pm 30.48\end{array}$ & $\begin{array}{c}0.148 \\
0.024 \\
<0.001 \\
0.190 \\
0.090\end{array}$ \\
\hline $\begin{array}{l}\text { Smoking status, } \%(n) \\
\text { Current smoker } \\
\text { Former smoker } \\
\text { Nonsmoker }\end{array}$ & $\begin{array}{c}33.5(177) \\
5.3(28) \\
61.2(324)\end{array}$ & $\begin{array}{l}33.6(49) \\
8.2(12) \\
58.2(85)\end{array}$ & $\begin{array}{c}28.4(82) \\
6.2(18) \\
65.4(189)\end{array}$ & $\begin{array}{l}0.297 \\
0.414 \\
0.297\end{array}$ & $\begin{array}{c}11.5(86) \\
1.5(11) \\
87.1(654)\end{array}$ & $\begin{array}{c}9.8(19) \\
1.0(2) \\
89.1(172)\end{array}$ & $\begin{array}{c}9.9(49) \\
2.6(13) \\
87.4(432)\end{array}$ & $\begin{array}{l}0.636 \\
0.219 \\
0.748\end{array}$ \\
\hline $\begin{array}{l}\text { Drinking status, } \%(n) \\
\text { Daily drinker } \\
\text { Occasional drinker } \\
\text { Nondrinker }\end{array}$ & $\begin{array}{c}18.3(97) \\
10.4(55) \\
71.3(377)\end{array}$ & $\begin{array}{l}16.4(24) \\
15.8(23) \\
67.8(99)\end{array}$ & $\begin{array}{c}13.8(40) \\
11.1(32) \\
75.1(217)\end{array}$ & $\begin{array}{l}0.256 \\
0.193 \\
0.251\end{array}$ & $\begin{array}{c}0.9(7) \\
0.5(4) \\
98.5(740)\end{array}$ & $\begin{array}{c}0.5(1) \\
1.0(2) \\
98.4(190)\end{array}$ & $\begin{array}{c}0.2(1) \\
0(0) \\
99.8(493)\end{array}$ & $\begin{array}{l}0.274 \\
0.129 \\
0.076\end{array}$ \\
\hline $\begin{array}{c}\text { Exercise status, } \%(n) \\
\text { High } \\
\text { Low } \\
\text { None }\end{array}$ & $\begin{array}{c}50.1(265) \\
14.9(79) \\
35.0(185)\end{array}$ & $\begin{array}{l}55.5(81) \\
14.4(21) \\
30.1(44)\end{array}$ & $\begin{array}{l}66.1(191)^{*, \#} \\
13.5(39) \\
20.4(59) *, \#\end{array}$ & $\begin{array}{c}<0.001 \\
0.855 \\
<0.001\end{array}$ & $\begin{array}{l}45.9(345) \\
16.6(125) \\
37.4(281)\end{array}$ & $\begin{array}{l}56.0(108) * \\
21.8(42) \\
22.3(43) *\end{array}$ & $\begin{array}{c}48.4(239)^{\#} \\
18.2(90) \\
33.4(165)^{\#}\end{array}$ & $\begin{array}{c}0.045 \\
0.247 \\
<0.001\end{array}$ \\
\hline Serum $M n, \mu g / L$ & $5.92(3.05,10.25)$ & $5.66(2.15,11.98)$ & $6.24(2.56,12.74)$ & 0.577 & $6.16(3.28,11.66)$ & $4.49(2.39,8.69) *$ & $\underset{\#}{5.56} \underset{(3.02,11.14)}{ }$ & 0.001 \\
\hline $\begin{array}{c}\text { Low, \%(n) } \\
\text { Normal, \%(n) } \\
\text { High, \%(n) }\end{array}$ & $\begin{array}{c}49.9(264) \\
48.2(255) \\
1.9(10)\end{array}$ & $\begin{array}{c}52.1(76) \\
47.3(69) \\
0.7(1)\end{array}$ & $\begin{array}{c}44.3(128) \\
53.3(154) \\
2.4(7)\end{array}$ & $\begin{array}{l}0.201 \\
0.316 \\
0.449\end{array}$ & $\begin{array}{c}49.0(368) \\
50.1(376) \\
0.9(7)\end{array}$ & $\begin{array}{c}58.0(112) \\
41.5(80) \\
0.5(1)\end{array}$ & $\begin{array}{c}50.2(248) \\
49.0(242) \\
0.8(4)\end{array}$ & $\begin{array}{l}0.080 \\
0.099 \\
0.851\end{array}$ \\
\hline
\end{tabular}


Table 2. Cont.

\begin{tabular}{|c|c|c|c|c|c|c|c|c|}
\hline \multirow[b]{2}{*}{ Variables } & \multicolumn{4}{|c|}{ Men } & \multicolumn{4}{|c|}{ Women } \\
\hline & $\begin{array}{l}\text { Normoglycemia } \\
\quad(n=529)\end{array}$ & $\begin{array}{l}\text { Prediabetes } \\
\quad(n=146)\end{array}$ & $\begin{array}{l}\text { Diabetes } \\
(n=289)\end{array}$ & $p$-Value & $\begin{array}{c}\text { Normoglycemia } \\
\quad(n=751)\end{array}$ & $\begin{array}{c}\text { Prediabetes } \\
\quad(n=193)\end{array}$ & $\begin{array}{l}\text { Diabetes } \\
(n=494)\end{array}$ & $p$-Value \\
\hline Disease & & & & & & & & \\
\hline Obesity, $\%(n)$ & $25.5(135)$ & $32.2(47)$ & $31.8(92)$ & 0.088 & $26.1(196)$ & $38.3(74) *$ & $34.6(171) *$ & $<0.001$ \\
\hline Hypertension, $\%(n)$ & $67.5(357)$ & $75.3(110)$ & $80.6(233)$ * & $<0.001$ & $64.7(486)$ & $74.1(143)$ * & $83.6(413) *$ *\# & $<0.001$ \\
\hline Stroke, $\%(n)$ & $6.2(33)$ & $5.5(8)$ & $9.3(27)$ & 0.183 & $2.3(17)$ & $1.0(2)$ & $4.9(24) * \#$ & 0.007 \\
\hline $\mathrm{CHD}, \%(n)$ & $11.5(61)$ & $7.5(11)$ & $11.4(33)$ & 0.368 & $16.8(126)$ & $13.5(26)$ & $19.4(96)$ & 0.157 \\
\hline
\end{tabular}

Prediabetes is defined by having fasting plasma glucose (FPG) levels $>110 \mathrm{mg} / \mathrm{dL}(6.1 \mathrm{mmol} / \mathrm{L}$ ) but $<126 \mathrm{mg} / \mathrm{dL}(7.0 \mathrm{mmol} / \mathrm{L}$ ); diabetes is defined by self-reported or FPG $>126 \mathrm{mg} / \mathrm{dL}$

$(7.0 \mathrm{mmol} / \mathrm{L})$; Abbreviations: SBP, systolic blood pressure; DBP, diastolic blood pressure; BMI, body mass index; TC, total cholesterol; TG, triglyceride; FPG, fasting plasma glucose; BUN, blood urea nitrogen; CRE, creatinine; CHD, coronary heart disease; Obtained by using ANOVA for continuous variables and chi-square for variables of proportion; All continuous variables were presented as means $\pm \mathrm{SD}$ or medians (interquartile ranges), and all categorical variables were presented as number (proportions); ${ }^{*}$ normoglycemia compare with prediabetes \& diabetes; ${ }^{\#}$ prediabetes compare with diabetes $(p<0.05)$ 


\subsection{The Associations of Serum Mn Levels with Prediabetes and Diabetes}

After adjusting for age, sex, BMI, lifestyle factors, and health status, participants with serum Mn levels in the higher quartiles exhibited a significant trend towards having lower ORs for the presence of prediabetes ( $p=0.005$, Table 3). When the higher quartiles (Q2 and Q3) were compared to the lowest quartile, the ORs for dysglycemia were 0.723 (95\% CI: 0.569-0.918) and 0.704 (95\% CI: 0.554-0.895), respectively (Table 3). No relationships were found between serum Mn levels and diabetes.

Table 3. Adjusted odds ratios (95\% confidence interval) of the association between serum Mn quartiles and dysglycemia (prediabetes and diabetes).

\begin{tabular}{|c|c|c|c|c|c|}
\hline \multirow{2}{*}{ Variables } & \multicolumn{4}{|c|}{ Serum Mn Quartiles } & \multirow{2}{*}{$\begin{array}{l}p \text { for } \\
\text { Trend }\end{array}$} \\
\hline & Q1 & Q2 & Q3 & Q4 & \\
\hline \multicolumn{6}{|c|}{ Prediabetes } \\
\hline Mn, $\mu \mathrm{g} / \mathrm{L}$ (serum level) & $0 \sim 2.858$ & $2.858 \sim 5.332$ & 5.332 9.676 & $9.676 \sim 46.000$ & \\
\hline Number at risk & 404 & 405 & 405 & 405 & \\
\hline Number with prediabetes & 110 & 77 & 78 & 74 & \\
\hline Crude & 1.000 & $0.610(0.438,0.849)$ & $0.630(0.453,0.876)$ & $0590(0.423,0.824)$ & 0.003 \\
\hline Model A & 1.000 & $0.607(0.434,0.849)$ & $0.633(0.454,0.883)$ & $0.607(0.433,0.851)$ & 0.005 \\
\hline Model B & 1.000 & $0.625(0.446,0.877)$ & $0.619(0.442,0.867)$ & $0.614(0.437,0.863)$ & 0.005 \\
\hline \multicolumn{6}{|c|}{ Diabetes } \\
\hline $\mathrm{Mn}, \mu \mathrm{g} / \mathrm{L}$ (serum level) & $0 \sim 2.952$ & $2.952 \sim 5.534$ & $5.534 \sim 10.384$ & $10.384 \sim 46.340$ & \\
\hline Number at risk & 515 & 517 & 515 & 516 & \\
\hline Number with diabetes & 205 & 179 & 180 & 219 & \\
\hline Crude & 1.000 & $0.801(0.622,1.031)$ & $0.813(0.631,1.046)$ & $1.115(0.870,1.429)$ & 0.387 \\
\hline Model A & 1.000 & $0.800(0.619,1.035)$ & $0.806(0.623,1.043)$ & $1.141(0.887,1.469)$ & 0.316 \\
\hline Model B & 1.000 & $0.824(0.627,1.082)$ & $0.762(0.579,1.003)$ & $1.150(0.881,1.501)$ & 0.413 \\
\hline \multicolumn{6}{|c|}{ Prediabetes + Diabetes } \\
\hline Mn, $\mu \mathrm{g} / \mathrm{L}$ (serum level) & $0 \sim 2.836$ & $2.836 \sim 5.429$ & $5.429 \sim 10.092$ & $10.092 \sim 46.340$ & \\
\hline Number at risk & 601 & 601 & 600 & 600 & \\
\hline Number with dysglycemia & 309 & 257 & 263 & 293 & \\
\hline Crude & 1.000 & $0.706(0.562,0.886)$ & $0.737(0.588,0.926)$ & $0.902(0.719,1.131)$ & 0.463 \\
\hline Model A & 1.000 & $0.708(0.562,0.892)$ & $0.738(0.586,0.929)$ & $0.916(0.728,1.152)$ & 0.547 \\
\hline Model B & 1.000 & $0.723(0.569,0.918)$ & $0.704(0.554,0.895)$ & $0.909(0.717,1.153)$ & 0.413 \\
\hline
\end{tabular}

Mn: manganese; ORs were determined from logistic regression analyses for the quartiles of serum manganese, comparing participants with dysglycemia (prediabetes and/or diabetes) to those with normoglycemia; Crude: no adjustment; Model A: adjusted for age, sex, body mass index (BMI), smoking status, drinking status, exercise status; Model B: additionally adjusted for blood pressure (BP), triglyceride (TG), total cholesterol (TC), duration of diabetes, antidiabetic medication use and history of hypertension, stroke, and coronary heart disease (CHD); Adjusted odds ratio; $95 \% \mathrm{CI}$ in parentheses.

Table 4 shows the crude and adjusted associations of serum Mn levels (quartiles) with prediabetes and diabetes according to sex. In the crude analysis, women exhibited a lower prevalence of prediabetes with increasing serum Mn levels ( $p$ for trend $=0.009$ ). After adjusting for age, BMI, smoking status, drinking status, and exercise status (Model A), the ORs for prediabetes according to serum Mn quartiles among women were 1.000, 0.622 (95\% CI: 0.353-1.097), 0.471 (95\% CI: 0.263-0.842), and 0.426 (95\% CI: 0.232-0.784) ( $p$ for trend $=0.02$ ). These results were unchanged when we adjusted for the other confounding factors in Model B. When the third Mn quartile was compared to the lowest quartile, the ORs in Model B for diabetes and dysglycemia were 0.675 (95\% CI: $0.474-0.962)$ and 0.668 ( $95 \%$ CI: $0.489-0.913)$, respectively. However, there was no linear association between serum Mn levels and diabetes or dysglycemia among women ( $p$ for trend $>0.05$ ). 
Table 4. Adjusted odds ratios (95\% confidence interval) of the association between serum Mn quartiles and dysglycemia (prediabetes and/or diabetes) according to sex.

\begin{tabular}{|c|c|c|c|c|c|c|c|c|c|c|}
\hline \multirow{2}{*}{ Variables } & \multicolumn{5}{|c|}{ Men } & \multicolumn{5}{|c|}{ Women } \\
\hline & Q1 & Q2 & Q3 & Q4 & $p$ for Trend & Q1 & Q2 & Q3 & Q4 & $p$ for Trend \\
\hline \multicolumn{11}{|c|}{ Prediabetes } \\
\hline Serum Mn $(\mu \mathrm{g} / \mathrm{L})$ & $0 \sim 2.52$ & $2.52 \sim 5.12$ & $5.13 \sim 9.85$ & $9.86 \sim 46.00$ & & $0 \sim 2.91$ & $2.91 \sim 5.65$ & $5.65 \sim 11.06$ & $11.06 \sim 15.49$ & \\
\hline Number at risk & 168 & 169 & 169 & 169 & & 236 & 236 & 236 & 236 & \\
\hline Number with prediabetes & 49 & 27 & 36 & 34 & & 62 & 49 & 42 & 40 & \\
\hline Crude & 1.000 & $0.462(0.272,0.784)$ & $0.657(0.400,1.080)$ & $0.612(0.370,1.011)$ & 0.126 & 1.000 & $0.578(0.332,1.006)$ & $0.473(0.266,0.839)$ & $0.383(0.211,0.699)$ & 0.009 \\
\hline Model A & 1.000 & $0.455(0.267,0.776)$ & $0.657(0.398,1.083)$ & $0.615(0.370,1.021)$ & 0.137 & 1.000 & $0.622(0.353,1.097)$ & $0.471(0.263,0.842)$ & $0.426(0.232,0.784)$ & 0.020 \\
\hline Model B & 1.000 & $0.463(0.269,0.798)$ & $0.639(0.383,1.065)$ & $0.614(0.365,1.031)$ & 0.134 & 1.000 & $0.773(0.498,1.200)$ & $0.602(0.382,0.947)$ & $0.603(0.381,0.953)$ & 0.015 \\
\hline \multicolumn{11}{|c|}{ Diabetes } \\
\hline Serum Mn $(\mu \mathrm{g} / \mathrm{L})$ & $0 \sim 2.92$ & $2.92 \sim 5.74$ & $5.74 \sim 10.30$ & $10.30 \sim 46.34$ & & $0 \sim 2.95$ & $2.95 \sim 5.45$ & $5.45 \sim 10.38$ & $10.38 \sim 26.38$ & \\
\hline Number at risk & 204 & 205 & 204 & 205 & & 311 & 312 & 311 & 311 & \\
\hline Number with diabetes & 76 & 55 & 69 & 89 & & 130 & 124 & 109 & 131 & \\
\hline Crude & 1.000 & $0.618(0.406,0.939)$ & $0.867(0.578,1.302)$ & $1.267(0.852,1.883)$ & 0.104 & 1.000 & $0.918(0.667,1.264)$ & $0.751(0.543,1.039)$ & $1.013(0.737,1.393)$ & 0.868 \\
\hline Model A & 1.000 & $0.642(0.418,0.987)$ & $0.860(0.567,1.303)$ & $1.283(0.854,1.925)$ & 0.113 & 1.000 & $0.905(0.654,1.254)$ & $0.734(0.527,1.020)$ & $1.020(0.738,1.410)$ & 0.808 \\
\hline Model B & 1.000 & $0.659(0.414,0.992)$ & $0.850(0.544,1.326)$ & $1.361(0.884,2.096)$ & 0.086 & 1.000 & $0.989(0.701,1.396)$ & $0.675(0.474,0.962)$ & $1.007(0.714,1.420)$ & 0.649 \\
\hline \multicolumn{11}{|c|}{ Prediabetes + Diabetes } \\
\hline Serum Mn $(\mu \mathrm{g} / \mathrm{L})$ & $0 \sim 2.80$ & $2.83 \sim 5.58$ & $5.61 \sim 10.20$ & $10.22 \sim 46.34$ & & $0 \sim 2.86$ & $2.86 \sim 5.33$ & $5.34 \sim 9.99$ & $9.99 \sim 26.38$ & \\
\hline Number at risk & 241 & 241 & 241 & 241 & & 358 & 361 & 360 & 359 & \\
\hline $\begin{array}{l}\text { Number with } \\
\text { dysglycemia }\end{array}$ & 122 & 87 & 105 & 121 & & 186 & 171 & 159 & 171 & \\
\hline Crude & 1.000 & $0.551(0.383,0.793)$ & $0.753(0.526,1.078)$ & $0.984(0.688,1.406)$ & 0.664 & 1.000 & $0.832(0.621,1.115)$ & $0.732(0.545,0.981)$ & $0.841(0.627,1.128)$ & 0.171 \\
\hline Model A & 1.000 & $0.574(0.397,0.832)$ & $0.760(0.528,1.095)$ & $0.992(0.690,1.425)$ & 0.671 & 1.000 & $0.823(0.611,1.108)$ & $0.726(0.539,0.978)$ & $0.852(0.633,1.147)$ & 0.206 \\
\hline Model B & 1.000 & $0.572(0.388,0.844)$ & $0.744(0.509,1.088)$ & $0.987(0.677,1.439)$ & 0.728 & 1.000 & $0.888(0.652,1.208)$ & $0.668(0.489,0.913)$ & $0.847(0.622,1.153)$ & 0.117 \\
\hline
\end{tabular}

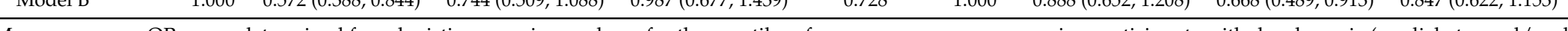
to those with normoglycemia; Crude: no adjustment; Model A: adjusted for age, body mass index (BMI), smoking status, drinking status, exercise status; Model B: additionally adjusted for blood pressure (BP), triglyceride (TG), total cholesterol (TC), duration of diabetes, antidiabetic medication use, and history of hypertension, stroke, and coronary heart disease (CHD); Adjusted odds ratio; 95\% CI in parentheses. 
Among men, the crude ORs for prediabetes according to serum Mn quartile were 1.000, 0.462 (95\% CI: 0.272-0.784, $p=0.004), 0.657$ (95\% CI: 0.400-1.080, $p=0.098$ ), and 0.612 (95\% CI: 0.370-1.011, $p=0.055)$; the OR was significantly lower for the second Mn quartile. The adjusted ORs for prediabetes in the second Mn quartile were 0.455 (95\% CI: 0.267-0.776, $p=0.004)$ for Model A and 0.463 (95\% CI: $0.269-0.798, p=0.003$ ) for Model B (vs. the first quartile). Similar results were observed in the diabetes and dysglycemia (prediabetes or diabetes) analyses, with the crude OR for the second Mn quartile being the lowest (OR for diabetes: $0.618,95 \%$ CI: $0.406-0.939, p=0.024$; OR for dysglycemia: 0.551 , 95\% CI: 0.383-0.793, $p=0.001$ ). These lower ORs for diabetes and dysglycemia remained significant in both Model A and Model B (Table 4). There was no linear association between serum Mn levels and prediabetes or diabetes among men ( $p$ for trend $>0.05$ ).

We also assessed the relationship between log-transformed Mn levels and the prevalences of prediabetes and diabetes. In the crude model, increasing log-transformed Mn levels exhibited a significant inverse relationship with the prevalence of prediabetes ( $p=0.003$ for the total population; $p=0.040$ for men; and $p=0.033$ for women). Even after the adjustment in the final model (Model B), the significant inverse relation remained unchanged (total population: OR: $0.705,95 \%$ CI: $0.555-0.896$, $p=0.004$; men: OR: 0.673, 95\% CI: 0.465-0.975, $p=0.036$; women: OR: 0.732, 95\% CI: 0.531-0.974, $p=0.046)$. However, no inverse relationship between log-transformed $\mathrm{Mn}$ levels and diabetes or dysglycemia was observed in the models.

\section{Discussion}

This study examined the associations of serum Mn levels with prediabetes and diabetes among community-dwelling adults who were $\geq 60$ years old. Although several studies have investigated the relationships between levels of trace elements and diabetes, few studies have evaluated the relationship between serum Mn levels and diabetes [11,12]. Moreover, no studies have evaluated the relationship between serum Mn levels and prediabetes according to sex. Thus, the strength of this large community-based study is that we were able to adjust for several confounding factors, and our results suggest that high serum Mn levels are independently associated with a lower prevalence of prediabetes among older women. Moderate serum Mn levels (the second quartile) were independently associated with lower prevalences of prediabetes and diabetes among older men.

Four small-scale cross-sectional studies have evaluated the relationships between levels of trace elements (including plasma Mn) and diabetes among American [30], Austrian [31], Turkish [32], and Czech [12] individuals. However, these studies did not find a statistically significant relationship between Mn levels and diabetes. In contrast, several other epidemiological studies have found an inverse relationship between Mn levels and diabetes among Russian [13], Pakistani [14], Italian [15], Nigerian [33], and Korean [11] individuals. Additionally, several other cross-sectional studies have found a positive association between Mn levels and diabetes among Russian [16], Mexican [17], and Turkish [34] individuals. Only two of these studies evaluated the general population [11,12], and only one study targeted older people [12]. Thus, our study provides the first data regarding the associations of Mn levels with prediabetes and diabetes among older Chinese individuals. Our results indicate that there is an inverse linear relationship between Mn levels and prediabetes among older women (but no linear relationship between Mn levels and diabetes), and that an association exists between moderate Mn levels and prediabetes/diabetes among older men. Although the reason for this discrepancy remains unclear, it is likely that differences in regions, dietary habits, race, and age may contribute. For example, age is associated with declining Mn levels [25]. Moreover, food is the main source of Mn in the general population [35] and different foods contain different levels of Mn, dietary manganese intake levels range from $1.38 \mathrm{mg} /$ day to $6.8 \mathrm{mg} /$ day, based on country-specific differences dietary patterns [36-40].

In the present study, women exhibited a negative linear association of serum Mn levels with prediabetes, although this association was not observed among men. This difference is likely related to circulating Mn levels being regulated by complex homeostatic mechanisms, and these levels may 
vary according to sex, age, and other variables [25]. Some studies have revealed that estrogen use did not affect Mn status among adult women [41], and hormonal replacement therapy does not influence serum Mn status among postmenopausal women [42]. Another study of dietary Mn intake revealed that women absorb significantly more Mn (vs. men) from a diet with adequate Mn [26]. That study also found that the biological half-life of Mn was significantly shorter in women, compared to men. These findings suggest that men and women exhibit differences in Mn absorption and metabolism, which may partially explain the sex differences that we observed in the present study. In addition, a Spanish study showed that inadequate Mn intake might favor insulin resistance among girls, which suggests that ensuring adequate Mn intake may help prevent insulin resistance and type 2 diabetes among girls [43]. Therefore, these results indicate that altered Mn metabolism is more important for women, who might be more sensitive to Mn deficiency, and we speculate that the sex differences may be related to differences in Mn metabolism, rather than hormonal differences. Nevertheless, further research is needed to explain the sex-specific differences in the association of Mn levels with prediabetes and diabetes.

We did not detect any sex-specific differences in serum Mn levels for participants with normoglycemia, prediabetes, or diabetes, although several other studies have reported higher blood Mn levels among women (vs. men) [44-46]. However, the Canadian and South Korean studies did not clarify the differences between men and women who were $\geq 60$ years old [44,46]. In contrast, the Swedish study reported a small difference between men and women who were $\geq 70$ years old (7.3 mg/L vs. $7.9 \mathrm{mg} / \mathrm{L}$, respectively) [45]. Nevertheless, our results are consistent with those from a Czech study [12]. Therefore, we speculate that sex-specific differences in serum Mn levels may also be related to age, which is supported by an American study that found sex-specific differences in blood Mn levels decreased with age [25]. In addition, a Korean study found that blood Mn levels decreased in postmenopausal women compared to that in premenopausal women after adjusting for various factors [47], which suggests that hormone may partly explain the insignificant sex-specific differences in blood Mn levels between men and postmenopausal women in our study.

We only observed a linear association between serum Mn levels and prediabetes among women, although all of the participants were $\geq 60$ years old and may have had diabetes for an extended period of time. Chronic diabetes is typically managed using medication and diet control, which might affect Mn metabolism. Thus, diabetes-associated changes in diet and medication may confound analyses of Mn levels and diabetes, which could also explain the different conclusions from the various studies. In contrast, individuals with prediabetes are not yet receiving hypoglycemic medication or dietary adjustment, which may provide a more accurate state for evaluating changes in Mn metabolism.

Appropriate levels of $\mathrm{Mn}$ are required for insulin synthesis and secretion, while Mn deficiency can lead to poor glucose metabolism [48]. High serum Mn levels might also be involved in oxidative stress protection and increase insulin secretion. Some studies have found that blood Mn levels range from $1.6 \mu \mathrm{g} / \mathrm{L}$ to $62.5 \mu \mathrm{g} / \mathrm{L}$, with changes according to sex, age, race/ethnicity and other blood mineral levels $[24,49]$. An animal study also revealed that manganese superoxide dismutase metalation and activity in normal mice can be augmented using Mn supplementation, and that Mn treatment increased insulin secretion to improve glucose tolerance during periods of dietary stress [50]. Nevertheless, $\mathrm{Mn}$ is a trace element with unclear essential and adverse effects, as excess $\mathrm{Mn}$ is related to neurotoxic effects [51], although the negative effects are only induced after ingestion of contaminated food or water [15]. There is only adequate intake in China ( $4.5 \mathrm{mg} /$ day), as the tolerable upper intake limit is $11 \mathrm{mg} /$ day [52], which may be related to insufficient information regarding $\mathrm{Mn}$ requirements. Thus, optimal Mn intake levels are not known, and our results might suggest the sex differences should be considered when recommending dietary intake in future guidelines.

This study had several limitations. First, the cross-sectional design precludes any conclusions regarding the causality or direction of the associations between serum Mn levels and prediabetes or diabetes. Second, although we adjusted for various confounding factors, we cannot exclude the possibility that the associations of serum Mn levels with prediabetes or diabetes are affected by other 
dietary factors, medications (such as HRT), and/or Mn supplementation. In addition, we cannot adjust for $\mathrm{HbA} 1 \mathrm{c}$ levels, as these data were not obtained during the health screening. Third, we did not consider other trace elements (e.g., iron or zinc) that can affect Mn levels, and some studies have found that individuals with low iron status have higher blood Mn levels [43,53]. This relationship may also explain the sex-specific differences in Mn metabolism. Therefore, a well-designed prospective study is needed to validate our findings, and we hope to provide additional data in our future studies.

\section{Conclusions}

This study revealed that serum Mn levels are likely associated with prediabetes and diabetes among older Chinese people. For example, higher serum Mn levels were independently associated with a lower prevalence of prediabetes among older community-dwelling women, which suggests that a Mn-rich diet may be useful in this population. Furthermore, we found that moderate serum Mn levels were associated with lower prevalence of prediabetes and diabetes among older men, which suggests that a normal dietary intake of Mn may be beneficial in this population. Nevertheless, there may be sex-specific roles for $\mathrm{Mn}$ in prediabetes and diabetes, and prospective studies of older adults are needed to confirm these findings.

Acknowledgments: This research was supported by the National Science and Technology Support Program (No. 2012BAI02B02).

Author Contributions: X.W. and G.H. conceived and designed the experiments; X.W., Z.L., Y.L., H.C. and M.Z. performed the study; X.W., Z.L., and M.Z. performed the statistical analysis; M.Z. and G.L. performed the manganese measurements; W. L. participated in the interpretation and presentation of the results. X.W. and G.H. wrote the paper; and X.W. and G.H. have primary responsibility for the final content. All authors read and approved the final manuscript.

Conflicts of Interest: The authors declare no conflict of interest.

\section{References}

1. Berry, C.; Tardif, J.C.; Bourassa, M.G. Coronary heart disease in patients with diabetes: Part I: Recent advances in prevention and noninvasive management. J. Am. Coll. Cardiol. 2007, 49, 631-642. [CrossRef] [PubMed]

2. Wild, S.; Roglic, G.; Green, A.; Sicree, R.; King, H. Global prevalence of diabetes: Estimates for the year 2000 and projections for 2030. Diabetes Care 2004, 27, 1047-1053. [CrossRef] [PubMed]

3. Xu, Y.; Wang, L.; He, J.; Bi, Y.; Li, M.; Wang, T.; Wang, L.; Jiang, Y.; Dai, M.; Lu, J.; et al. Prevalence and control of diabetes in Chinese adults. JAMA 2013, 310, 948-959. [CrossRef] [PubMed]

4. Cheng, Q.; Sun, H.X.; Ye, F.L.; Wang, G.; Ling, H.W.; Chen, S.D.; Jiang, G.X. Dementia among elderly in Shanghai suburb: A rural community survey. J. Alzheimer's Dis. 2014, 39, 883-889.

5. Fraga, C.G. Relevance, essentiality and toxicity of trace elements in human health. Mol. Asp. Med. 2005, 26, 235-244. [CrossRef] [PubMed]

6. Aschner, J.L.; Aschner, M. Nutritional aspects of manganese homeostasis. Mol. Asp. Med. 2005, 26, 353-362. [CrossRef] [PubMed]

7. Malecki, E.A.; Huttner, D.L.; Greger, J.L. Manganese status, gut endogenous losses of manganese, and antioxidant enzyme activity in rats fed varying levels of manganese and fat. Biol. Trace Elem. Res. 1994, 42, 17-29. [CrossRef] [PubMed]

8. Bond, J.S.; Failla, M.L.; Unger, D.F. Elevated manganese concentration and arginase activity in livers of streptozotocin-induced diabetic rats. J. Biol. Chem. 1983, 258, 8004-8009. [PubMed]

9. Korc, M. Manganese action on pancreatic protein synthesis in normal and diabetic rats. Am. J. Physiol. 1983, 245, G628-G634. [PubMed]

10. Candilish, D.J. Minerals. J. Am. Coll. Nutr. 2000, 17, 286-310.

11. Koh, E.S.; Kim, S.J.; Yoon, H.E.; Chung, J.H.; Chung, S.; Park, C.W.; Chang, Y.S.; Shin, S.J. Association of blood manganese level with diabetes and renal dysfunction: A cross-sectional study of the Korean general population. BMC Endocr. Disord. 2014, 14, 24. [CrossRef] [PubMed] 
12. Rambousková, J.; Krsková, A.; Slavíková, M.; Cejchanová, M.; Wranová, K.; Procházka, B.; Cerná, M. Trace elements in the blood of institutionalized elderly in the Czech Republic. Arch. Gerontol. Geriatr. 2013, 56, 389-394. [CrossRef] [PubMed]

13. Kosenko, L.G. Concentration of trace elements in blood of patients with diabetes mellitus. Fed. Proc. Transl. Suppl. 1965, 24, 237-238. [PubMed]

14. Kazi, T.G.; Afridi, H.I.; Kazi, N.; Jamali, M.K.; Arain, M.B.; Jalbani, N.; Kandhro, G.A. Copper, chromium, manganese, iron, nickel, and zinc levels in biological samples of diabetes mellitus patients. Biol. Trace Elem. Res. 2008, 122, 1-18. [CrossRef] [PubMed]

15. Forte, G.; Bocca, B.; Peruzzu, A.; Tolu, F.; Asara, Y.; Farace, C.; Oggiano, R.; Madeddu, R. Blood metals concentration in type 1 and type 2 diabetics. Biol. Trace Elem. Res. 2013, 156, 79-90. [CrossRef] [PubMed]

16. Lisun-Lobanova, V.P. Trace elements (manganese, copper, zinc) in patients with diabetes mellitus. Zdravookhr. Beloruss. 1963, 9, 49-53. [PubMed]

17. Flores, C.R.; Puga, M.P.; Wrobel, K.; Garay Sevilla, M.E.; Wrobel, K. Trace elements status in diabetes mellitus type 2: Possible role of the interaction between molybdenum and copper in the progress of typical complications. Diabetes Res. Clin. Pract. 2011, 91, 333-341. [CrossRef] [PubMed]

18. Mowé, M.; Bøhmer, T.; Kindt, E. Reduced nutritional status in an elderly population ( $>70$ years) is probable before disease and possibly contributes to the development of disease. Am. J. Clin. Nutr. 1994, 59, 317-324. [PubMed]

19. Beletate, V.; El Dib, R.P.; Atallah, A.N. Zinc supplementation for the prevention of type 2 diabetes mellitus. Cochrane Database Syst. Rev. 2007, 24. [CrossRef]

20. Nathan, D.M.; Davidson, M.B.; De Fronzo, R.A.; Heine, R.J.; Henry, R.R.; Pratley, R.; Zinman, B.; American Diabetes Association. Impaired fasting glucose and impaired glucose tolerance: Implications for care. Diabetes Care 2007, 30, 753-759. [CrossRef] [PubMed]

21. Twigg, S.M.; Kamp, M.C.; Davis, T.M.; Neylon, E.K.; Flack, J.R.; Australian Diabetes Society; Australian Diabetes Educators Association. Prediabetes: A position statement from the Australian Diabetes Society and Australian Diabetes Educators Association. Med. J. Aust. 2007, 186, 461-465. [PubMed]

22. Haase, H.; Overbeck, S.; Rink, L. Zinc supplementation for the treatment or prevention of disease: Current status and future perspectives. Exp. Gerontol. 2008, 43, 394-408. [CrossRef] [PubMed]

23. Department of Noncommunicable Disease Surveillance. Part 1. Diagnosis and classification of diabetes mellitus. In Definition, Diagnosis and Classification of Diabetes Mellitus and Its Complications: Report of a WHO Consultation; World Health Organization: Geneva, Switzerland, 1999.

24. Ngueta, G.; Kengne, A.P. Low-level environmental lead exposure and dysglycemia in adult individuals: Results from the Canadian health and measure survey 2007-2011. Biol. Trace Elem. Res. 2016. [CrossRef] [PubMed]

25. Oulhote, Y.; Mergler, D.; Bouchard, M.F. Sex- and age-differences in blood manganese levels in the U.S. general population: National health and nutrition examination survey 2011-2012. Environ. Health 2014, 13. [CrossRef] [PubMed]

26. Finley, J.W.; Johnson, P.E.; Johnson, L.K. Sex affects manganese absorption and retention by humans from a diet adequate in manganese. Am. J. Clin. Nutr. 1994, 60, 949-955. [PubMed]

27. Onat, A.; Hergenç, G.; Keleş, I.; Doğan, Y.; Türkmen, S.; Sansoy, V. Sex difference in development of diabetes and cardiovascular disease on the way from obesity and metabolic syndrome. Metabolism 2005, 54, 800-808. [CrossRef] [PubMed]

28. Monterrosa, A.E.; Haffner, S.M.; Stern, M.P.; Hazuda, H.P. Sex difference in lifestyle factors predictive of diabetes in Mexican-Americans. Diabetes Care 1995, 18, 448-456. [CrossRef] [PubMed]

29. Paek, K.W.; Chun, K.H. Sex difference of type 2 diabetes affected by abdominal obesity versus overall obesity. Yonsei Med. J. 2010, 51, 850-856. [CrossRef] [PubMed]

30. Walter, R.M., Jr.; Uriu-Hare, J.Y.; Olin, K.L.; Oster, M.H.; Anawalt, B.D.; Critchfield, J.W.; Keen, C.L. Copper, zinc, manganese, and magnesium status and complications of diabetes mellitus. Diabetes Care 1991, 14, 1050-1056. [CrossRef] [PubMed]

31. Serdar, M.A.; Bakir, F.; Haşimi, A.; Celik, T.; Akin, O.; Kenar, L.; Aykut, O.; Yildirimkaya, M. Trace and toxic element patterns in nonsmoker patients with noninsulin-dependent diabetes mellitus, impaired glucose tolerance, and fasting glucose. Int. J. Diabetes Dev. Ctries. 2009, 29, 35-40. [CrossRef] [PubMed] 
32. Ekmekcioglu, C.; Prohaska, C.; Pomazal, K.; Steffan, I.; Schernthaner, G.; Marktl, W. Concentrations of seven trace elements in different hematological matrices in patients with type 2 diabetes as compared to healthy controls. Biol. Trace Elem. Res. 2001, 79, 205-219. [CrossRef]

33. Adewumi, M.T.; Njoku, C.H.; Saidu, Y.; Abubakar, M.K.; Shehu, R.A.; Bilbis, L.S. Serum chromium, copper and manganese levels of diabetic subjects in Katsina, Nigeria. Asian J. Biochem. 2007, 2, 284-288.

34. Ekin, S.; Mert, N.; Gunduz, H.; Meral, I. Serum sialic acid levels and selected mineral status in patients with type 2 diabetes mellitus. Biol. Trace Elem. Res. 2003, 94, 193-201. [CrossRef]

35. Williams, M.; Todd, G.D.; Roney, N.; Crawford, J.; Coles, C.; McClure, P.R.; Garey, J.D.; Zaccaria, K.; Citra, M. Toxicological Profile for Manganese; Agency for Toxic Substances and Disease Registry: Atlanta, GA, USA, 2012.

36. Wang, L.D. Comprehensive Report of Chinese National Nutrition and Health Survey in 2002; People's Medical Publishing House: Beijing, China, 2005.

37. Leblanc, J.C.; Guérin, T.; Noël, L.; Calamassi-Tran, G.; Volatier, J.L.; Verger, P. Dietary exposure estimates of 18 elements from the 1st French Total Diet Study. Food Addit. Contam. 2005, 22, 624-641. [CrossRef] [PubMed]

38. Turconi, G.; Minoia, C.; Ronchi, A.; Roggi, C. Dietary exposure estimates of twenty-one trace elements from a Total Diet Study carried out in Pavia, Northern Italy. Br. J. Nutr. 2009, 101, 1200-1208. [CrossRef] [PubMed]

39. Rubio, C.; Gutiérrez, A.J.; Revert, C.; Reguera, J.I.; Burgos, A.; Hardisson, A. Daily dietary intake of iron, copper, zinc and manganese in a Spanish population. Int. J. Food Sci. Nutr. 2009, 60, 590-600. [CrossRef] [PubMed]

40. Kim, E.Y.; Bae, Y.J.; Kim, S.J.; Choi, M.K. Estimation of manganese daily intake among adults in Korea. Nutr. Res. Pract. 2008, 2, 22-25. [CrossRef] [PubMed]

41. Davis, C.D.; Greger, J.L. Longitudinal changes of manganese-dependent superoxide dismutase and other indexes of manganese and iron status in women. Am. J. Clin. Nutr. 1992, 55, 747-752. [PubMed]

42. Bureau, I.; Anderson, R.A.; Arnaud, J.; Raysiguier, Y.; Favier, A.E.; Roussel, A.M. Trace mineral status in post menopausal women: Impact of hormonal replacement therapy. J. Trace Elem. Med. Biol. 2002, 16, 9-13. [CrossRef]

43. Rodríguez-Rodríguez, E.; Bermejo, L.M.; López-Sobaler, A.M.; Ortega, R.M. An inadequate intake of manganese may favour insulin resistance in girls. Nutr. Hosp. 2011, 26, 965-970. [PubMed]

44. Clark, N.A.; Teschke, K.; Rideout, K.; Copes, R. Trace element levels in adults from the west coast of Canada and associations with age, gender, diet, activities, and levels of other trace elements. Chemosphere 2007, 70, 155-164. [CrossRef] [PubMed]

45. Olsén, L.; Lind, P.M.; Lind, L. Gender differences for associations between circulating levels of metals and coronary risk in the elderly. Int. J. Hyg. Environ. Health 2012, 215, 411-417. [CrossRef] [PubMed]

46. Lee, J.W.; Lee, C.K.; Moon, C.S.; Choi, I.J.; Lee, K.J.; Yi, S.M.; Jang, B.K.; Yoon, B.J.; Kim, D.S.; Peak, D.; et al. Korea National Survey for Environmental Pollutants in the Human Body 2008: Heavy metals in the blood or urine of the Korean population. Int. J. Hyg. Environ. Health 2012, 215, 449-457. [CrossRef] [PubMed]

47. Lee, B.K.; Kim, Y. Effects of menopause on blood manganese levels in women: Analysis of 2008-2009 Korean National Health and Nutrition Examination Survey data. Neurotoxicology 2012, 33, 401-405. [CrossRef] [PubMed]

48. Nicoloff, G.; Mutaftchiev, K.; Strashimirov, D.; Petrova, C. Serum manganese in children with diabetes mellitus type 1. Diabetol. Croat. 2003, 33, 47-51.

49. Bocca, B.; Madeddu, R.; Asara, Y.; Tolu, P.; Marchal, J.A.; Forte, G. Assessment of reference ranges for blood Cu, $\mathrm{Mn}, \mathrm{Se}$ and $\mathrm{Zn}$ in a selected Italian population. J. Trace Elem. Med. Biol. 2011, 25, 19-26. [CrossRef] [PubMed]

50. Lee, S.H.; Jouihan, H.A.; Cooksey, R.C.; Jones, D.; Kim, H.J.; Winge, D.R.; McClain, D.A. Manganese supplementation protects against diet-induced diabetes in wild type mice by enhancing insulin secretion. Endocrinology 2013, 154, 1029-1038. [CrossRef] [PubMed]

51. Mergler, D.; Baldwin, M. Early manifestations of manganese neurotoxicity in humans: An update. Environ. Res. 1997, 73, 92-100. [CrossRef] [PubMed]

52. Chinese Nutrition Society. Chinese Dietary Reference Intakes; Science Press: Beijing, China, 2013.

53. Kim, Y.; Lee, B.K. Iron deficiency increases blood manganese level in the Korean general population according to KNHANES 2008. Neurotoxicology 2011, 32, 247-254. [CrossRef] [PubMed]

(C) 2016 by the authors; licensee MDPI, Basel, Switzerland. This article is an open access article distributed under the terms and conditions of the Creative Commons Attribution (CC-BY) license (http:/ / creativecommons.org/licenses/by/4.0/). 